\title{
FINE RESOLUTION BRIGHTNESS DISTRIBUTION OF THE VISIBLE ZODIACAL LIGHT
}

\author{
S. M. KWON ${ }^{1,2}$, S. S. HONG ${ }^{1}$, J. L. WEINBERG ${ }^{2}$, AND N. Y. MISCONI ${ }^{2}$ \\ ${ }^{1}$ Department of Astronomy, Seoul National University, 151-742, KOREA \\ ${ }^{2}$ Space Astronomy Laboratory, University of Florida, U.S.A.
}

\begin{abstract}
Applying time-dependent corrections of the atmospheric diffuse light to the observed night sky brightness, we have determined brightness of the zodiacal light over the region $40^{\circ} \leq \lambda-\lambda_{\odot} \leq 320^{\circ}$ and $-20^{\circ} \leq \beta \leq 20^{\circ}$. The resulting map of equal brightness contours has an angular resolution of two degrees, and exhibits east-west and north-south asymmetries.
\end{abstract}

\section{Introduction}

For the purpose of reducing the zodiacal light from night sky observations, the major sources of the sky brightness may be classified (cf. Weinberg 1963) into the following four categories: the bright stars(BS) that are resolved as individual stars by a given telescope, the integrated starlight(IS) including the diffuse Galactic light, the zodiacal light(ZL) of our interest, and the airglow(AG) emitted by the earth upper atmosphere. Because of the scattering by atmospheric constituents, the telescope's field of view intercepts not only the directly transmitted light of all the four sources but also the diffusely scattered light of the latter three extended sources. Lack of accurate information about the IS distribution, difficulties in correcting for the diffusely scattered components, and uncertain nature of the AG time-variation all made it practically impossible to obtain, from single night observations of night sky, a fine resolution map of the ZL distribution. Careful analyses of the night sky observations accumulated over many seasons/years have given us a smoothed-out distribution of the ZL (Dumont 1965; Dumont and Sanchez 1975; Levasseur-Regourd and Dumont 1980), and Pioneer 10/11 space probes have actually measured the IS over almost entire sky (Toller 1981). On the basis of the space measurements and the smoothed-out ZL, a self-consistent empirical method has recently been developed for making the time-dependent corrections of the atmospheric diffuse light (Kwon, Hong and Weinberg 1991). With this method, we will analyze the single night observations of night sky brightness, and determine the ZL brightness over an extended area of sky. The resulting distribution of the ZL will be presented in the form of equal brightness contours with a $2^{\circ}$ resolution.

\section{Observational Data and Calibration}

From archives of the night sky observations by Weinberg and Mann, we selected the observations over one complete night, August 21/22 1968, at Mt. Haleakala, Hawaii. The sky was scanned repeatedly over the entire $360^{\circ}$ range of azimuth at 8 fixed zenith distances $45^{\circ}(05) 80^{\circ}$ by the photometer attached to a telescope of 1.85-radius field of view. After each set of 8 almucantar scans, they also scanned the entire northern meridian from zenith to horizon. During the night, 11 sets of 8 almucantar and 1 meridian scans were obtained for each wavelength of $5080 \AA$ and $5300 \AA$, 183

A.C. Levasseur-Regourd and H. Hasegawa (eds.), Origin and Evolution of Interplanetary Dust, 183-186. (c) 1991 Kluwer Academic Publishers, Printed in Japan. 


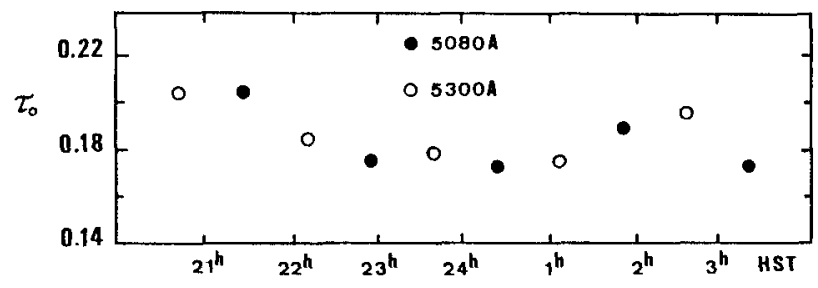

Fig 1 - Time-variation of the extinction optical depth of the atmosphere at zenith. The relative amplitude of the variation is about $15 \%$.

making 198 scans altogether. The 22 meridian scans have provided the means for making time- and position-dependent corrections of the atmospheric diffuse light (Kwon, Hong and Weinberg 1991), and the remaining 176 almucantar scans will be analyzed in this paper. (Further details of the observations may be found from Weinberg and Mann [1967].)

Contrary to the most of the previous studies, in our scheme of data analysis, such reduction parameters as the zenith extinction optical depth $\tau_{0}$ of the atmosphere, the effective field of view (FOV) of the telescope, and the calibration factor $C$ are all simultaneously determined from the same set of observational data, from which the ZL brightness is to be reduced. This ensures us of an internal consistency of the reduction scheme, and also reduces the level of errors in the resulting ZL brightness.

Ninety three bright stars are identified with distinct peaks in the scan profiles (cf. the top curve in Fig 2). The maximum chart deflection $R$ of a star with magnitude $m_{\lambda}$ at wavelength $\lambda$ and the calibration factor $C$ in units of $S_{10}(V)_{G 2 V}$ per unit deflection are related by the relation $C \cdot R \cdot F O V=\eta 10^{0.4\left(10-m_{\lambda}\right)} \cdot \exp \left[-\tau_{0} x\right]$, where $x$ is the airmass, and $\eta$, the conversion factor from $S_{10}(\lambda)$ to $S_{10}(V)_{G 2 V}$, is 1.20 for $5080 \AA$ and 1.05 for $5300 \AA$. Since $m_{\lambda}$ 's for all the stars can be known by interpolating $\mathrm{V}$ and $\mathrm{B}-\mathrm{V}$ values of the stars and $x$ 's for the stars are determined from their zenith distances at the moment of observation, the relations for a number of stars comprise a set of simultaneous equations for the two unknowns $\tau_{0}$ and $C \cdot F O V$. Application of the least squares fit to the data determines the best values for $\tau_{0}$ and $C \cdot F O V$.

In order to take into account for the changing atmospheric conditions, we divided the 11 sets for one wavelength into 5 sub-groups, and $\tau_{0}$ is determined for each group with the value for $C \cdot F O V$ being fixed at its average for all the scans. The resulting $\tau_{0}$ 's for the two wavelengths are plotted in Fig 1 as a function of Hawaii standard time. The means of the time-dependent $\tau_{0}$ 's are 0.183 for $5080 \AA$ and 0.173 for $5300 \AA$, and the relative amplitude of the time-variation amounts to about $15 \%$. If one ignores the time-variation of $\tau_{0}$, significant errors would come into the derived brightness of the ZL.

\section{Eliminations of the BS, IS and Atmospheric Diffuse Light}

From the General Catalogue of 33342 Stars (Roman and Warren 1985), the Nicolet Homogeneous UBV Catalogue (Nicolet 1978), the Bright Star Catalogue (Hoffleit 1982), and the U.S. Naval Observatory Photoelectric Catalogue (Blanco et al. 1968), we have collected all the positional and photometric information about the resolved stars, 8372 in total, that are brighter than 6.5 mag in the visual, and stored them in a single data-base. We search the data-base for those bright stars that come into the given FOV along the scan path, and synthesize the brightness profile due to the stars. For the IS, we also synthesize the profile by using the Pioneer observations. Sum of the two profiles is then compared with the observed profile, graphically on a computer screen. An example of the synthetic profiles is given in Fig 2 by the dashed curve. By adjusting the trial values for $C$ and FOV until the comparison gives satisfactory results, particularly over the regions of very bright stars and the Galactic plane, we finally determine accurate values for $C$ and FOV separately.

By subtracting the synthetic profile that is obtained with the best values of $\tau_{0}$, FOV and $C$, from 

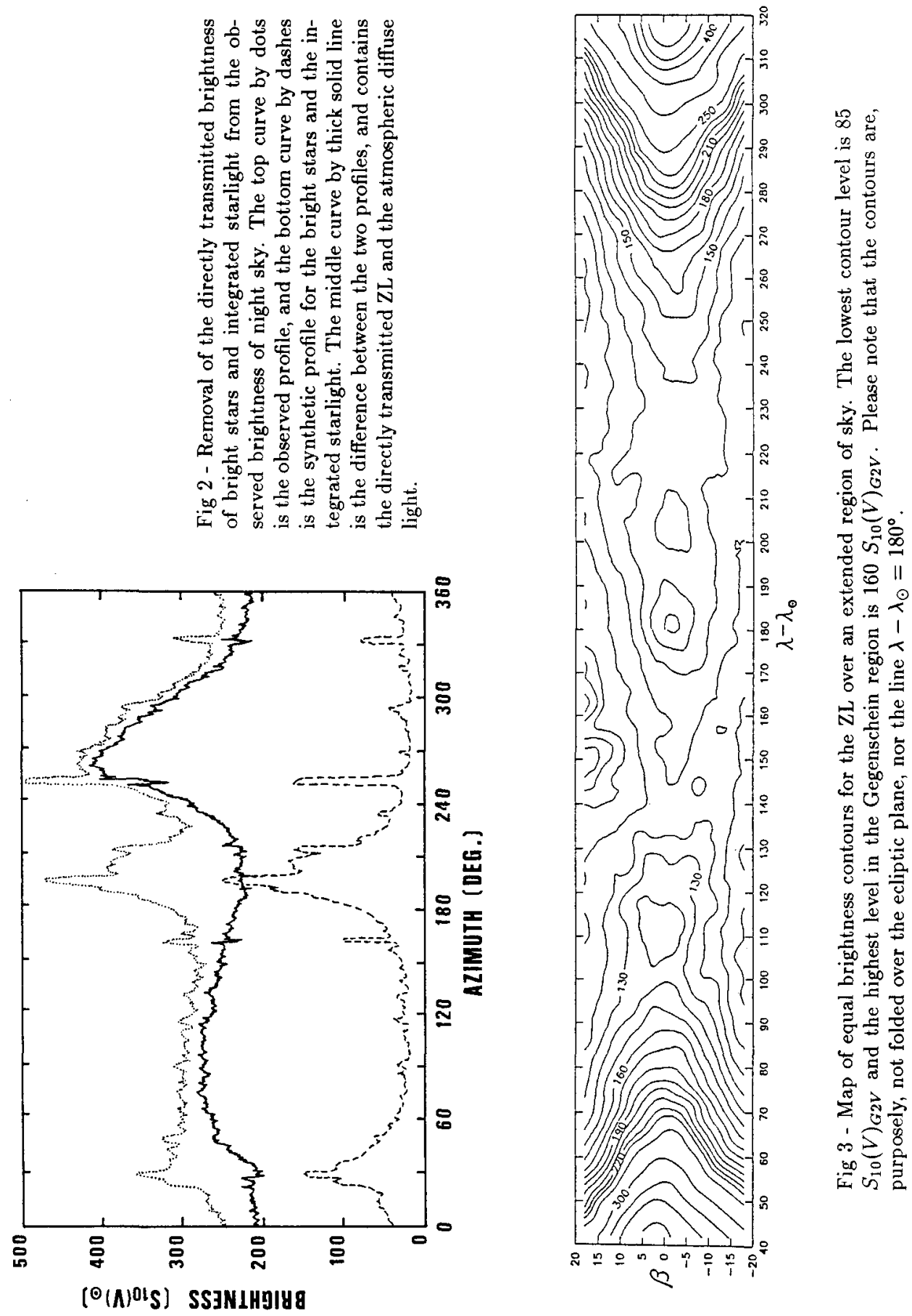
the observed profile, we removed the directly transmitted contributions of the BS and IS from the observed brightness. The thick solid line in Fig 2 represents the profile for the residual brightness. The residual still contains, in addition to the $\mathrm{ZL}$, the atmospheric diffuse light (ADL), which is composed of the directly transmitted AG and the diffusely scattered components of the IS and ZL.

The ADL brightness even at a fixed zenith distance varies with time over a night. The Barbier's relation of two parameters have been modified to describe the dependence of the ADL brightness upon zenith distance. By the use of time dependent values for the two parameters, Kwon, Hong and Weinberg (1991) are able to describe the temporal and spatial variations of the ADL with the modified relation of Barbier. Time-dependent values of the ADL are then removed from the residual brightness profiles to finally obtain the ZL brightness along the paths of almucantar scans.

\section{A Fine Resolution Map of the ZL Brightness}

For each wavelength, we have produced a two dimensional $\left(\lambda-\lambda_{\odot}, \beta\right)$ map of isophotal ZL contours with an angular resolution of two degrees on average (here $\lambda$ meaning the ecliptic longitude). An internal consistency of our reduction methodology has been demonstrated by good agreements in the contour morphology and the brightness between the two maps for $5080 \AA$ and $5300 \AA$. This study has reduced the errors in the $\mathrm{ZL}$ brightness from the previous level of higher than $15 \%$ to the present level of $10 \%$ or less. The two maps are stacked upon each other, and the combined map is shown in Fig 3.

The brightness distribution of the ZL clearly exhibits asymmetries between the morning side $\left(\lambda-\lambda_{\odot}>180^{\circ}\right)$ and evening side $\left(\lambda-\lambda_{\odot}<180^{\circ}\right)$, and also between the north and south of the ecliptic. The position of peak brightness in the Gegenschein is located below the ecliptic plane. These features of asymmetry are interpreted as a consequence of the disalignment of the surface of densest dust concentration with respect to the ecliptic. The $\mathrm{ZL}$ distribution reduced from the single night observations not only has the information about the symmetry plane(s) but also sets up the observational criteria for competing models of the zodiacal dust cloud. The ZL map presented in this study is hoped to provide a platform for probing the zodiacal cloud in three dimensions.

SSH and SMK were supported by the Basic Science Research Institute Program, Korean Ministry of Education. At an earlier stage of this study, SMK, JLW and NYM were partially supported by the US AFOSR.

\section{REFERENCES}

Blanco, V. M., Demers, S., Douglass, G. G., and Fitzerald, M. P. 1968, Photoelectric Catalogue, Magnitudes and Colours of Stars in the UBV and $U_{c} B V$ Systems, ser. 2, vol. 21 (U.S. Naval Observatory:Washigton, D.C.)

Dumont, R. 1965, Ann. d'Astrophys., 28, 265

Dumont, R., and Sanchez, F. 1975, Astr. Ap., 38, 397

Hoffleit, D. 1982, The Bright Star Catalogue (Yale:New Haven)

Kwon, S. M., Hong, S. S., and Weinberg, J. L. 1991, in this volume

Levasseur-Regourd, A. C. and Dumont, R. 1980, Astr. Ap., 84, 227

Nicolet, B. 1978, Astr. Ap. Suppl., 34, 1

Roman, G. N., and Warren, W. H. Jr. 1985, Documentation for the Machine-Readable Version of the General Catalogue of 39342 Stars for the Epoch 1950, NSSDC/WDC-A-R\&S 83-07

Toller, G. N. 1981, Ph. D. Thesis, State University of New York at Stony Brook

Weinberg, J. L. 1963, Ph. D. Thesis, University of Colorado

Weinberg, J. L., and Mann, H. M. 1967, in Proceedings of the Symposium on the Zodiacal Light and the Interplanetary Medium, NASA SP-150, ed. J. L. Weinberg, p.3 